\title{
COMPLEX EMERGENCE AND THE LIVING ORGANIZATION: AN EPISTEMOLOGICAL FRAMEWORK FOR BIOLOGY
}

\author{
Published in Synthese (2012), 185(2): 215-232
}

Penultimate version: the final publication is available at Springer Link: http://link.springer.com/article/10.1007\%2Fs11229-010-9722-6

\author{
Leonardo Bich \\ Postgraduate School of Anthropology and Epistemology of Complexity, University of Bergamo, \\ P.le S.Agostino 2, 24129 Bergamo, Italy.
}

\begin{abstract}
In this article an epistemological framework is proposed in order to integrate the emergentist thought with systemic studies on biological autonomy, which are focused on the role of organization. Particular attention will be paid to the role of the observer's activity, especially: (a) the different operations he performs in order to identify the pertinent elements at each descriptive level, and (b) the relationships between the different models he builds from them. According to the approach sustained here, organization will be considered as the result of a specific operation of identification of the relational properties of the functional components of a system, which do not necessarily coincide with the intrinsic properties of its structural constituents. Also, an epistemological notion of emergence-that of "complex emergence"-will be introduced, which can be defined as the insufficiency, even in principle, of a single descriptive modality to provide a complete description of certain classes of systems. This integrative framework will allow us to deal with two issues in biological and emergentist studies: (1) distinguishing the autonomy proper of living systems from some physical processes like those of structural stability and pattern generation, and (2)
\end{abstract}


reconsidering the notion of downward causation not as a direct or indirect influence of the whole on its parts, but instead as an epistemological problem of interaction between descriptive domains in which the concept of organization proposed and the observational operations related to it play a crucial role.

Keywords: autopoiesis; biological autonomy; complex emergence; downward causation; organization; pattern generation.

\author{
"We are inheritors of categorized \\ knowledge; therefore we inherit a world \\ view that consists of parts strung together, \\ rather than of wholes regarded through \\ different sets of filters" (S. Beer, 1980)
}

\title{
1. Introduction
}

The biological processes of realization of a living unity that exhibits a certain degree of autonomy with respect to its environment are characterized by the production of a stable shape. An operation of self-distinction from the medium due to the generation of a physical boundary (Varela et al. 1974; Maturana and Varela, 1980) gives rise to a separation between the internal and the external environment of the organism.

These, however, do not constitute the only phenomena of production of higher-level patterns - stable in time and with respect to external perturbations - that we can identify in the natural world. Similar properties are also exhibited by some physico-chemical systems like the processes of molecular self-assembly, Benard's cells and chemical clocks, explained by the thermodynamics of dissipative structures (Nicolis and Prigogine 1977; Prigogine and Stengers 1979). Similar results can be obtained also in the computational domain through appropriate simulations. For this reason, the very notions of pattern and of structural stability, although they constitute a relevant aspect 
of the biological phenomenology, cannot be considered as the elements that allow us to capture the specificity of the living with respect to physical and artificial systems.

All these kinds of processes show some properties that can be ascribed to an intuitive notion of emergence, considered as a generic novelty. In all these cases, in fact, the properties that we consider as stable, regular, or "ordered" can be identified at the higher level of the unities but not at the level of their components. But also a generic notion of emergence like this does not provide any conceptual tool in order to understand the specificity of those unities that characterize the biological domain.

According to these remarks, which can be the role of the concept of emergence in the study of biological systems? A way to face this problem can be looked for by analyzing the modality of constitution of the living as an integrated whole and by focusing on the relation between the higher-level unity and its constituents. This problem is connected to two crucial issues in theoretical biology and systems theory, which show an intertwining of theoretical and epistemological aspects at the very foundations of these disciplines. The first one consists in the attempt to characterize what can be considered as the basic living system. It is the problem of defining what makes any organism a unity belonging to a certain class, and which specificities make it different from other kinds of unities that we can distinguish in other domains, like the physical or the chemical ones. The second issue concerns the role of the scientific observer in recognizing these particular kinds of unities and in elaborating theoretical and formal models of them.

The purpose of this article is to provide a possible answer to the question posed above by facing these two aspects. In doing so I will propose a synthesis between the emergentist thought and the tradition of studies on biological autonomy, which produced its most mature theoretical expression in the theory of autopoiesis. This was elaborated in the early seventies by Humberto Maturana and Francisco Varela (Varela et al. 1974; Maturana and Varela 1980, 1984), who developed more in depth some of the ideas about life and cognition also proposed by Jean Piaget (1967).

At the same time I will assume a specific epistemological approach, also based on Maturana and Varela's thought. It is focused on the observer's activity in his interaction with natural systems, rather than on the objective properties of those systems themselves. In other words, I will analyze the concept of emergence and the theoretical 
models of living systems from the point of view of the operations of construction of descriptions and of their reciprocal relations.

In adapting this theoretical and epistemological framework to the insights coming from the study of emergent systems, I will also show how it diverges in some crucial aspects from what can be referred to as the tradition of "self-organization" (Banzhaf 2002; Damiano 2009). The concepts of autonomy and self-organization are often used as synonymous with the consequence of bringing some confusion into the debate about emergence and the living (Bich 2008), and this analysis will provide some conceptual tools in order to distinguish between them.

This article is structured in five steps. In the first one I will analyze the concept of biological autonomy, focusing on the distinction between organization and structure, which is crucial in Maturana and Varela's thought. A living system will be defined as a unity on the relational level of its organization rather than on the level of its physicochemical constituents. In the second step I will outline an epistemological framework useful for analyzing the autonomy of the living from an emergentist point of view. In the following two parts of the paper I will consider two different classes of emergent processes which, from the epistemological point of view, can be defined in terms of unpredictability and non-deducibility. The aim will be to show that, at the level of the basic conceptual assumptions, self-organizing and autonomous systems involve two different conceptions of higher-level emergent "unities;" also, that they implicitly entail two different heuristics, respectively characterized by bottom-up and top-down identifications of the relevant components. In the last step I will analyze the inter-level relations implied by this framework, providing an epistemological solution to the problem of downward causation.

\section{Investigating biological autonomy}

The starting point of the studies on biological autonomy consists in the attempt to answer the fundamental question of biology - What is life? - by re-posing it on the pertinent level of description (Bich and Damiano 2007). The traditional definitions of life based on the properties of metabolism, reproduction and evolvability are in fact 
focused respectively on the chemical level and on the levels of populations and species. As a result, in order to answer this question they miss, and implicitly presuppose, the primary level: that of the individual living unity. In fact it is the living that provides the conditions under which the metabolic processes take place, and that reproduces, thus giving rise to the process of evolution.

If we focus instead on the level of the individual living unity, the question about life can be re-formulated in the following way: What kind of unity characterizes the continuous and intertwined flux of production of components that we recognize as realizing a living system?

This conceptual step is of paramount importance with respect to the problem of the characterization of the specificity of the biological domain. It represents a further development of the initial analysis of biological autonomy considered as a partial independence of a system from environmental perturbations (external autonomy), characteristic of the first cybernetics (Wiener 1948). According to the autopoietic approach this property, which is shared and can be simulated by a wide class of natural and artificial systems, needs to be grounded in the specific characteristics of the generative processes that realize the system as a living organism (internal autonomy). In such a way biological autonomy can be defined more precisely. In fact the "external" generic property of partial independence from the environment - that in living systems consists in self-confinement due to the production of a membrane and in the capability to actively respond to environmental perturbations not specified by the nature of the perturbations themselves - can be explained as the consequence of the internal properties of self-production of components and of self-maintenance of the whole network of processes.

If we shift to this internal point of view, some difficulties arise when we try to capture what makes a living system a unity and what kind of invariant order we can ascribe to it. We are dealing, in fact, with a system which is characterized by a continuous change at the level of its components and which nevertheless maintains itself. In machines, in simulations in the computational domain, and in processes of physico-chemical self-assembly, the constituents are arranged in an order that is observable according to some spatial properties. In living organisms, on the other hand, the chemical components are mixed together in a continuous flux of processes. As a 
consequence, the higher-level unitary order we can ascribe to them is not identifiable according to the positions of the components; it is not localizable. This particular order, to which we referred elsewhere as "order in the nothing" (Bich and Damiano 2008), needs to be identified on a different and more abstract level of description than the one concerning the constituents and their reciprocal positions.

In order to identify the invariant aspect that characterizes a living system with respect to its continuous internal flux of changes, the autopoietic biology introduced by Maturana and Varela (Varela et al. 1974; Maturana and Varela 1980, 1984) uses a peculiar descriptive modality. It is a relational qualitative description based on the systemic thesis that what is characteristic of an organism is in fact not the single physical processes which take place in it, but instead the way these are related in order to produce and maintain the integrated biological unity they belong to. As a consequence, what is conserved invariant (?) in this continuous change is not to be found at the structural physico-chemical level, but on a more abstract, though complementary, level of description: the level of the relations between these processes, that is, the level of their organization. By "organization" we mean here the topology of relations that allows us as scientific observers to identify a system as a unity belonging to a certain class, in this case the class of living systems. The identification of this level of description is at the basis of the definition of the living as possessing an autopoietic organization, that is, a closed network of processes of production, transformation and destruction of components, which:

(1) through their interactions and transformations recursively realize and regenerate the same complex of processes that produces them;

(2) constitute the system as a concrete unity in the space in which they exist, by establishing its boundaries and so specifying its domain of existence with respect to its environment (Varela et al. 1974; Maturana and Varela 1980).

In such a way the properties of self-production, self-maintenance and self-distinction from an environment are made dependent on the particular shape assumed by the network of relations that constitute the organization of the living system: a selfreferential circle expressed by the notion of organizational "closure" (Piaget 1967; Varela et al. 1974; Varela 1979; Maturana and Varela 1980), whose formal 
modelization had already been attempted by Robert Rosen since the late fifties (Rosen 1958, 1991).

At this step at least two levels of description are introduced. The first, structure, concerns the material constituents of the systems, characterized according to their intrinsic properties: They are described in physico-chemical terms. The second, organization, concerns the set of relations that define the unitary identity of the system. At this level the constituents are characterized according to their relational properties and the pertinent description of the system is a relational one (Bich and Damiano 2008). According to this scenario, organization can be considered as a new higher-order unitary level. Its processual unity as a network of relations is physically realized by the processes involving the structural constituents, and is maintained invariant with respect to the lower-level molecular transformations that take place between them. At these two levels of description we can identify two different kinds of "closure," which make the living system a unity separated and autonomous with respect to its environment. The first is a physical one, at the level of structure: the boundary of the system. The second is a functional and relational one at the level of the organization: the autopoietic selfreferential network.

It is therefore necessary to make explicit the specific connection that involves organization and its emergence in the biological domain. The purpose is to characterize the autonomy of the biological unities, the living systems, by finding a middle way that avoids both naive holism and the mere additivity of aggregative unities. In the first case the wholes are not processual but, on the contrary, they are already given and not analyzable. They can be studied only through an input-output description. In the second case the autonomy of the higher level is epiphenomenal and can be traced back to a single-level description of the behavior of the structural components. The aim is, thus, to be able to conceive a kind of process that involves two or more descriptive levels, characterized by an effective autonomy with respect to the others. In other words, it consists in showing the double nature of the process of integration of a unity that is effective and permanent in its global identity, but never complete. It is, in fact, always realized by the variety of its lower-level changing structure. This double nature can be expressed by the descriptive complementarity between structure and organization. 
In such a framework the concept of emergence makes it possible to conceive a possible distinction between an aggregative unity and an integrated one. It seems indispensable from the point of view of biological autonomy, as it allows characterization of the living as a "system," a global unity which cannot be considered as a mere resultant, an epiphenomenon, but as an effective process whose presence is at the basis of the discontinuities we observe in the natural world between the physical and the biological domains. The concept of organization, on the other hand, becomes crucial in order to understand the generative mechanism of this unitary and integrated character that is typical of the living.

From this point of view, the relation between these concepts forms the conceptual basis of the distinction between the invariance that characterizes autonomy and the stability of a structural pattern. As will be shown in more detail in the following sections, these two kinds of invariance are generated according to different modalities and are realized in different descriptive domains: the organizational one for autonomy, and the structural one for pattern stability. In the latter case the unity exhibits a certain shape that is recognized by the observer thanks to its temporal stability, but it requires an external source of reference. In the former, however, the invariance is not just a spatial and temporal regularity. It has an effective role in the system itself and can be identified by an observer in the same domain in which the system specifies it through its own operations. What is crucial here is a functional invariance rather than a structural persistence in time (Varela 1979, 56; Anderson and Stein 1985, 447).

\section{The epistemological framework}

The connection between emergence and organization had already been pointed out by the first analyses of the concept of emergence elaborated by the British Emergentism. This is particularly evident in Conwy Lloyd Morgan's formulation of the concept of relatedness:

The discussion of relatedness [...] requires the consideration of the terms in relation within any given field of relatedness, and of the relations of these terms. Relatedness, in my sense of the word, includes both; not the terms only; not the 
relations only. [...] What is supervenient at any emergent stage of the evolutionary progress is a new kind of relatedness - new terms in new relations hitherto not in being. (Lloyd Morgan 1923, 19)

According to this view terms and relations co-emerge and co-specify, as any new emergent level is instantiated. The terms, which can be considered as the functional components of the system, are inseparable from the relations (the organization) they take part in. As a consequence they cannot be characterized according to their intrinsic properties; they are defined instead by their relational ones.

This inseparability between terms and relations is the theoretical tool that allows Lloyd Morgan to account for the specificities of the interactions that take place in biological systems with respect to the trivially mechanistic ones. These interactions do not determine a mere rearrangement of the components. Instead they have an effective role because they do entail a difference in the connected terms with respect to a description of them as independent. Furthermore, the very properties of the terms depend on and can be defined according to the interactions they enter. In such a scenario emergence can be characterized as the constitution of a new field of relatedness between the elements of a process, whose properties are not intrinsic but are defined by the same field they constitute. This is the reason for the non-reductive descriptive gaps between levels characteristic of certain classes of systems, especially the biological ones.

This co-emergence, where at any new level the components are characterized according to their relational properties, shows some affinities with the concept of organization proposed by the autopoietic theory. One of the main differences is that Lloyd Morgan's analysis follows an ontological approach that encounters some difficulties. In fact it introduces a proliferation of levels of reality without providing an explanation of their generation or of their reciprocal relations that does not give rise to contradictions (Kim 1992, 1998).

The difficulties encountered by the ontological approach depend on the fact that if we admit a descriptive gap between levels, due to the instantiation of an emergent process, it is not possible to follow a traditional description that builds one level on the other without leaving unexplained any residual aspect (property or behavior) of the process. If we do not want to recur to vitalistic hypotheses, we risk remaining entrapped 
in an oscillation between continuity and discontinuity. A possible way of avoiding these problems can be found by facing this issue from an epistemological standpoint. This means shifting the attention into the cognitive domain of the observer, and considering the problem from the point of view of his operations and descriptions.

According to this framework emergence is to be considered as a relation between descriptions or models of natural systems and not between properties of an objective reality in itself. The problem of the gap between levels, which leads to the impossibility of predicting or deducing the behavior of a system from that of its material components, can be considered as a problem concerning descriptions. As such it can be approached from an epistemological point of view. In such a way the relation between different levels ceases to be an ontological one; it is brought inside the domain of experience of the observer. The observer therefore assumes a crucial effective role at the operational level in the recognition and characterization of the phenomena of emergence.

This choice does not consist in a denial of the existence of reality, but it is just a shift of point of view from the world to the observer who experiences it. It has two main consequences. The first is that there is no need to look for a fundamental level of description, as the starting point of any level is constituted by the observer himself (Ceruti 1989; Bitbol 2007). The different levels, in fact, are not built on one another, but rather all of them depend primarily on the observer's operations. Secondly, the classical distinction between an ontological kind of emergence, which relies on the properties of an objective reality, and an epistemological one, which depends on the limits of the observer (Silberstein and McGeever 1999), loses its meaning inside this framework. In fact we are dealing here with relations between descriptions. As a consequence, both kinds of emergence -as well as reduction - can now be considered as observerdependent and, in some sense, epistemological. Still they are such in different ways, which need to be made explicit in the course of this analysis.

The epistemological approach assumed here is based on a constructivist framework (Ceruti 1989; Bich 2008; Damiano 2009). It is derived from the "biogenetic approach to cognition" (Lyon 2006) which was developed by Maturana and Varela (Varela 1979; Maturana and Varela 1980, 1984; Varela et al. 1991) and which constitutes an integral part of their theory of living systems. According to this framework the starting point of a scientific analysis is not reality, but the experience of the observer who interacts with 
it. The descriptions he produces are considered as deriving from the regularities he experiences in his domain of interactions. Therefore, all that is available to the scientific observer are his operations, which include his experiences, his descriptions, and the relations he instantiates between them.

In this article one special operation is considered. It is the primary and most fundamental one, and it consists in the distinction of a unity from its background (Spencer Brown 1969; Varela 1979; Maturana and Varela 1980, 1984). This operation, which constitutes a crucial element of the autopoietic theory, is fundamental because it defines the domain of investigation and the elements that are pertinent in it. It depends on the purpose and on the point of view of the observer, who specifies the identity of the observed system together with its domain of existence, that is, its medium or background.

According to the autopoietic theory at least two levels can be distinguished on a single unity, each one determining a domain of existence characterized by specific elements and properties (Maturana and Varela 1980). But in order to face the problem of emergence, and for the coherence of the whole framework, a third one needs to be introduced (Bich 2008, 2009). All of these levels are characterized by the identification of certain pertinent unities, but they can be classified according to the differences in their point of view and in the modality of the operation from which they result:

1) The first level corresponds to an external point of view with respect to the system. It is the level of the simple unity, considered as a whole, which is distinguished from the medium it interacts with. It is the first one to be identified, according to the purposes of the observer;

2) The second level corresponds to the internal point of view. It is the level of the composite unity characterized by the interactions between the functional components of the system. These are distinguished in relation to the system they integrate. This operation, which follows a top-down direction, specifies the descriptive domain of the organization of the system, and the components are characterized according to their relational properties;

3) The third one is the level of the material parts (or structural parts) of the system. They are characterized according to their intrinsic properties, because they are considered in isolation or, better, as distinguished from a generic background. 
They are identified through an operation that follows a bottom-up direction, and their proper descriptive domain is the structure of the system.

The difference between the last two levels does not depend on a whole-parts relationship as with respect to the first one, but on the direction of the operation of distinction: top-down for the functional components of the composite unity; bottom-up for the material parts. In this scenario the emergent organization of the system, which can be associated with Lloyd Morgan's relatedness, can be interpreted as the result of a specific observational operation, which focuses on a different descriptive level with respect to the one characteristic of the structural constituents. This operation consists in the identification of the relational properties of the functional components of the system, instead of the intrinsic properties of its material parts.

By assuming this epistemological framework, when a unity is identified together with its domains of existence the crucial aspect in order to define which kind of unity we are dealing with, and which kind of relative independence it exhibits with respect to its lower-level constituents, depends on the relation between these domains. Consequently, the very problem of emergence needs to be reconsidered too. From this standpoint it will consist in the possibility to express the properties of a domain in terms of those of another one; in other words in the possibility to make these levels and the relative observational operations coincide, at least in principle.

After having identified the different descriptive domains specified by the operations of distinctions and what is needed in order to define the identity of a system considered as an emergent unity, a further step is necessary. It consists in the assumption of a metalevel of description from which to consider the relations between the different domains, that is, to specify and analyze the notion of emergence in terms of relations between descriptions. Then the epistemological tools provided by this redefinition of emergence will be applied to the two different classes of models of natural processes that characterize the debate about what is a living unity: the generation of stable patterns, and autonomy.

An intuitive notion of emergence as mere novelty is in fact insufficient in order to distinguish between these two classes of phenomena of constitution of a unity. It fails in taking into account the different possible kinds of descriptive relations between levels. It is therefore necessary to specify, if possible, in which sense these natural processes 
can be considered emergent. To do so means to specify what is the novelty that characterizes the higher level and how we can consider it "new" with respect to the lower ones (Crutchfield 1994). According to the epistemological framework introduced here, this must be expressed in terms of the operations performed by the observer and of the relations between the descriptions that can be derived from them.

Two kinds of observational or descriptive relations allow a distinction between two different ways to characterize emergence (Crutchfield 1994; Pessa 1998):

1. Emergence as unpredictability of a phenomenon with respect to its initial conditions or to the behavior of some basic level elements;

2. Emergence as non-deducibility of a phenomenon with respect to the model that describes the initial dynamics of the system or the behavior of its basic level constituents.

The processes of pattern generation, among others, belong to the first class; autonomous processes belong to the second one. Both definitions concern some limits in the capability of an observer to construct complete descriptions of some types of natural systems, usually referred to as complex. But they differentiate with respect to each other according to the characteristics of these very epistemic limits they entail. The thesis been sustained according to which even if these phenomena of emergence entail some descriptive limits, they are still effective and, as such, they have also an ontological status, not just an epistemological one (Bedau 2008). According to the epistemological framework assumed here, however, the attention is shifted from reality to the cognitive domain of the observer, and in particular to the properties of his descriptions. From this point of view, thus, the two different characterizations of emergence concern models and their relations, and not objective properties of systems themselves.

In the following sections these two classes of emergent phenomena will be analyzed by making explicit three aspects that are missing in the naïve approach to emergence:

(a) The kind of novelty that characterizes the higher level;

(b) The observational limits connected to it and the related modalities of modelization;

(c) The inter-level relationships these phenomena entail, that is, the kind of upward and downward causation they specify. 


\section{Emergence as unpredictability: self-organizing systems}

To this class belong different kinds of phenomena, usually defined as self-organizing (Banzhaf 2002), like those studied by the thermodynamics of dissipative structures (thermodynamical emergence) or the computational simulations produced in the domain of artificial life (computational emergence). The characteristic shared by these processes consists in the production of what is recognized by an external observer as a unitary shape of higher level with respect to the initial elements: an ordered spatial configuration. It is in principle deducible from the model that describes the dynamics of the constituents of the systems, but it is not predictable due to the characteristics of the interactions between them.

There are two relevant aspects that make it possible to characterize these processes as emergent: the non predictability of the dynamics and the production of a global unitary pattern. The first one is relative to the properties of the descriptive models of the process, and it is strictly connected to the notion of "error" in the measurement of the initial conditions of the system under study (Rosen 1978). Given that measurement is the identification of an interval of numbers in the continuum, the impossibility-even in principle - to discriminate between values of the observables below a certain threshold of accuracy constitutes an intrinsic limit for the observer. As a consequence, for certain systems characterized for example by some non-linear interactions, even if a complete description of the dynamics is available, an infinite precision in the measurement of the initial conditions is required. The impossibility of attaining it determines the first aspect of novelty regarding these phenomena: the divergence between the predicted and the effectively observed behavior of the system after a certain amount of time.

It must be pointed out that this novelty does not entail an effective gap between different levels of description, because it does not necessarily require a new descriptive framework to be provided in a different domain. The possible behaviors of the system and its constituents are in fact determined a priori by the initial model: A complete definition of the variables of the state of the system and a complete description of the interactions between the system and its environment are in principle available. What 
appears as a novelty depends rather on the impossibility to choose a priori which possible behavioral path will be realized.

The second aspect of novelty concerns the generation of an ordered pattern, whose appearance is not predictable, as specified above, due to the characteristics of the dynamics that realize it. The basic idea-which constitutes an inheritance of the law of conversion of quantity into quality proposed by Friedrich Engels in 1883 (Engels 1952) - is that after crossing a certain threshold in a variable of a system, we witness the instantiation of a phenomenon which is qualitatively different with respect to the initial one. An example of these transitions is the generation of a visible ordered pattern, typical of phenomena belonging to the physical and the computational domains.

The problem with this conceptual model of generation of qualitatively different new levels is that it derives from an epistemological mistake. The nature of the differences between distinct observational levels - the constituents and the ordered higher-level unity-is not taken into consideration. The identification of the higher-order pattern, whose novelty is of a predictive kind, depends in fact on a difference in the observational scale and not in the observational domain. From the operational point of view the behavior of the system can be described by the initial model of the constituents without the need to provide a new descriptive domain within which to model the new qualitative aspects. Consequently the new unitary level in this case is just an epiphenomenon whose description as a whole, even if heuristically useful, is not required at the operational level.

These classes of phenomena exhibit what can be considered a weak form of emergence similar to the one proposed by Mark Bedau (2003). In this case, however, the unity is not considered autonomous but just aggregative, even if it entails an epistemic limit "in principle," concerning the process of measurement. Some autonomy, anyway, can be attributed to it for heuristic purposes.

According to the epistemological framework introduced in the previous section, the constituents that are pertinent in order to describe the system are identified through an operation of distinction of the third kind. In fact they consist in the material parts, which are characterized according to their intrinsic properties. In this case they coincide with the functional components derived from the second kind of distinction: There is no difference between the results of these two operations. 
Therefore, the higher level does not show any autonomy with respect to the lower ones. Its unitary order constitutes only a useful tool for skipping some descriptive steps by avoiding taking into consideration all the lower-level dynamics. The spatial configuration produced by the system in fact has no effective role with respect to the system and, consequently, does not provide a novelty in a proper sense. It is just recognized by an external observer according to some a priori categories, possibly geometrical ones, which are relevant for him (Crutchfield 1994) in order to be able to simplify his descriptions while facing systems characterized by very complicated structures and dynamics. From the point of view of an internal observer, however, the production and recognition of the pattern are not important for the description of the system's behavior, because this is already totally specified by the model describing the interaction of its material parts characterized according to their intrinsic properties.

These phenomena do not constitute a proper model of what characterizes the living as an effective autonomous and integrated unity. In fact if we take into consideration the process of construction of their descriptions and the characteristics of the resultant models, there is no effective generation of novel levels. The apparent wholeness of the configurations they produce depends only on the aggregative, even if complicate, interaction between the material parts of the system, without generating any observational gap from the operational point of view. The most complete, or ideal, form of knowledge of these systems is therefore still the complete initial description of their material parts distinguished from a generic background. However it must be pointed out that this approach distinguishes itself from the Laplacian ideal of complete "divine" knowledge, because it must take into account the intrinsic limits in the processes of measurement and as a consequence it will never provide even in principle complete predictability of the future behavior of the system.

According to these remarks these phenomena can be better defined as weakly supervenient than as emergent in a proper sense:

A weakly supervenes on $\mathrm{B}$ if and only if necessarily for any $\mathrm{x}$ and $\mathrm{y}$ if $\mathrm{x}$ and $\mathrm{y}$ share all properties in $\mathrm{B}$ then $\mathrm{x}$ and $\mathrm{y}$ share all properties in $\mathrm{A}$ - that is, indiscernibility with respect to $\mathrm{B}$ entails indiscernibility with respect to A. (Kim $1993,58)$ 
They are in fact characterized by some co-variance between, at least, two observational scales, even if not a trivial one. Their dynamics exhibit behaviors like that of "canalization" (Waddington 1968; Weiss 1969), which can be characterized as a many-to-one correspondence between the different scales. The higher level, though epiphenomenal, can be considered the resultant of manifold possible lower-level interactions.

This is the aspect that makes them interesting from an emergentist point of view as, according to these properties, they cannot be subjected to a functional reduction like the one proposed for example by Jaegwon Kim through the establishing of biunivocal interlevel correspondences (Kim 1997). So they require new strategies to be developed. A possible reductive solution-coherent with Bedau's framework of weak emergence (Bedau 2003) — consists in the computational simulation of the evolution of the system.

An implicit conception of modelization is entailed by the conceptual characterization of these phenomena. It holds that the observables necessary in order to describe the system are not defined on the level of the system as a whole but on that of its subsets, that is, of its material parts distinguished in isolation. Therefore the observed system is not the domain of the operation of construction of its models, but the range: It is not the starting point of the description but the result of a conceptual additive construction from its material constituents. Its models in fact derive from an operation of assembly of the models that describe its structural parts, following an exclusively bottom-up direction (Rosen 1991). Thus, the identification of the intrinsic properties of the constituents, and not the systemic properties of the whole, is primary.

If we take into consideration the remarks provided in this section, it is also necessary to point out a terminological issue. The expression 'self-organization', which is usually ascribed to this class of phenomena, refers here to a "structural organization" that comes out in the process of generation of a spatial order through self-aggregation. In fact in this context the two distinct descriptive levels, structure and organization, can be considered equivalent: The second and third operations of distinction, respectively of the functional components and of the material parts, are the converse of one another. Organization here, considered at the same level as structure, has a meaning which is different from the one that comes out of the definition given above, which entails the 
instantiation of a new abstract descriptive level, complementary with respect to the structural one.

The term 'self-organization' still maintains its historical validity as it involves a long tradition of studies in the domain of complex systems, and there would be no need to stop using it. However it is necessary to make it explicit that when we are using this expression in this context, we are referring to phenomena that could be better defined as "self-assembly" or "self-ordering" (Abel and Trevors 2006; Bich 2008).

\section{Complex emergence and autonomous systems}

Which kind of model of emergent phenomena accounts for the production of a novelty that has an effective role for the very system that produces it, and which is missing in the processes of pattern generation? In order to answer to this question it is necessary to analyze the second class of emergence which, from the descriptive point of view, instantiates a relation of non-deducibility.

In this class of phenomena the realization of a new system or the exhibition of a new behavior cannot be deduced, even in principle, from the model that describes the initial dynamics. As a consequence a new description is needed: a new model that cannot be reduced either to the initial one or to a more comprehensive one. This class of phenomena includes the spontaneous symmetry breaking described by quantum field theory (Anderson and Stein 1985; Humphreys 1997; Pessa 1998; Bitbol 2007) in physics. This kind of descriptive relation can also be applied to autopoietic and evolutive processes in the biological domain (Bich 2008), as it makes it possible to express a strong form of autonomy. Also, it provides a framework and a heuristic which can be useful in order to address some crucial problems, like structure and function in proteins (Rosen 1991) and the identification of the relevant components or complexes of processes in the unitary dynamics of biological systems.

Differently from the previous class of processes of production of novelty, in this case emergence is not relative to the accuracy of the observables. The descriptive limitation entailed in this context, although still of an epistemic nature, does not depend on the process of measurement of an observable, but instead on the relations between 
classes of observables that converge in different models. It can be defined as the insufficiency of a single model, and of the set of observables related to it, to describe a system. Consequently the model needs to be replaced, because the system exhibits to the observer new characteristics which were not present before, and which are thus invisible to the chosen observables that constitute the initial description.

This scenario is particularly coherent with the epistemological definition of complexity proposed by Rosen in the seventies:

To say that a system is complex [...] is to say that we can describe the same system in a variety of distinct ways [...]. Complexity then ceases to be an intrinsic property of a system, but it is rather a function of the number of separate descriptions required [...]. Therefore a system is simple to the extent that a single description suffices to account for our interaction with the system; it is complex to the extent that it fails to be true. (Rosen 1978, 112)

According to this definition, I chose to refer to this class of emergent phenomena by using the expression 'complex emergence' (Bich 2008, 2009), which accounts for both the importance of how the system is organized in different layers-which instantiate distinct and complementary descriptive domains - and the role of the observer who needs to recur to different modalities of description in order to account for them. With 'complex emergence' we therefore express the failure or the inadequacy of a single descriptive modality and the consequent necessity to move to new ones. In this sense it completes the shifting of meaning of the concept of emergence, from the expression of ontological properties of a system to the relation between different models that are needed in order to describe the experiences performed by an observer who interacts with it. It is in fact this very lack of a direct relationship between different descriptions of distinct domains that in this framework expresses more precisely the notion of emergence. Also, it provides a possible solution to some conceptual problems related to emergence, as we will see with respect to the issue of downward causation.

In processes exhibiting complex emergence the new level that is generated cannot be described in terms of the lower ones. Consequently, according to this epistemological framework, it can be characterized as an effective one, which imposes a fracture in the domain of descriptions established by an observer. In particular, with respect to the distinction made before, organization in this case belongs to a qualitatively different 
level with respect to the structural one; a level characterized by elements and properties that, in order to be described, necessitate the elaboration of new models.

Therefore, for an operational characterization of a system exhibiting complex emergence all three descriptive domains generated by an operation of distinction need to be taken into consideration. While in phenomena of pattern generation the levels of material parts and functional components can be considered as coincident, and the unity constitutes an epiphenomenon, in this class of emergent processes the descriptive levels are distinct and the lower one is not pertinent in the description of the other two. In other words the observational operations from which they derive are not equivalent. As a result, some autonomy can be ascribed to the level of organization with respect to the structural one. They belong to distinct descriptive domains. The difference between the domains of components (organization) and material parts (structure), characterized by specific models irreducible to one another, in fact is due to the different directions of the operations of distinction. They are respectively the top-down and bottom-up ones, which in this case are not just the inverse of one another.

In autonomous systems, in fact, the emergent level - the systemic unity-does not supervene on the intrinsic properties of the elements of the lower level, distinguished and characterized as independent from each other and from the unity they structurally realize. Since material parts and functional components do not coincide and are placed in different descriptive domains, we observe an effective gap between levels. As a consequence it is impossible to establish a direct relation between them. The unity, in fact, cannot be described through the interactions between the material parts. Yet it can be at least theoretically modeled from an analysis of the composite unity, as this one follows a top-down direction and its components depend on the relational higher level they realize.

According to these remarks, in the modelization of the system as a unity the relevant observables are identified on the system and not on its material constituents: The direction is top-down. It is the inverse of the previous case, as here the domain of the modeling operation is constituted by the very system, and the range by its components (Rosen 1991). Also, the descriptive gap between levels implies a further difference in the modelization from the processes of pattern generation: There is no direct correspondence between the organization of the composite unity (top-down direction) 
and the structure realized by the material parts (bottom up direction). The sets of models derived from these observational operations therefore provide different, though complementary, information about the system under study.

In physics phenomena of this kind have already been described in detail. The paradigmatic example is spontaneous symmetry breaking in quantum field theory (Anderson and Stein 1985), which Paul Humphreys (1997) analyzed in terms of the relation of fusion, and which has also been interpreted in epistemological terms (Pessa 1998; Bitbol 2007). The characteristic that makes this a clear example of a phenomenom of complex emergence, coherent with the framework proposed here, is that the material parts constitute only the initial constituents, which cannot be observed after the emergence of the higher unitary level. Also, the higher level and its functional components co-emerge (Bitbol 2007): The bosons in fact cannot be observed independently from the system that contains them, and consequently cannot be characterized as independent material constituents. As a result the unity can be considered to exhibit some autonomy with respect to its parts, as its properties do not supervene on those of its initial constituents.

In biology the situation is more complicated, and also involves a complex heuristic procedure that does not just consist in the immediate identification of the different descriptive domains. This is due to two main reasons. The first is the lack of formal models of the integrated dynamics of the minimal relational living unity. The second, which constitutes the main difference between biological processes and the physical ones outlined above, depends on the fact that we do not observe the emergence of new objects which exclude the initial ones. In biological systems, in fact, material parts and functional components can be observed simultaneously, even if through different kinds of observational operations and in different descriptive domains. A relational unity emerges in them, an effective unitary organization that specifies the conditions for its own realization by self-distinguishing from a background from both the functional and the structural points of view: not just from the latter. Its emergence is internally selfspecified, and as such it is not relevant only for an external observer. So the difficult challenge consists in identifying those functional relational components that co-emerge together with its unitary identity, and which do not coincide with the structural physicochemical constituents. 


\section{Downward causation}

Some further remarks needs to be provided about the inter-level relation between parts and whole in the two classes of emergent phenomena analyzed: the problem of downward causation. Following the classification proposed by Emmeche et al. (2000), the processes of pattern generation exhibit a weak form of downward causation. It is an epiphenomenic one, as the instantiation of the higher level does not imply an effective change inside the system in the behavior of its constituents, which would require the formulation of a new model.

For this reason it falls under the critics advanced by Kim through his Principle of Explanatory Exclusion (Kim 1998). In a "single-domain" conception of components in which material part and functional ones are coincident, the influence of the whole on the parts can be only epiphenomenic. Otherwise there would be a causal overdetermination: Everything is in fact already entailed at the lower level. Therefore the effective processes take place only at the "fundamental" level of description where all the processes are already completely defined - at least in principle - by the respective model.

In phenomena exhibiting complex emergence the scenario is different, for epistemological reasons. These processes, being effective, can be associated to a medium version of downward causation (Emmeche et al. 2000) to which to ascribe an operational role without involving a proliferation of ontological levels. But when we attempt to conceive this multilevel relationship, some conceptual problems come up (Bich 2008, 2009). They concern the possibility to conceive from an operational point of view a causal power of the whole on its parts while these, for the same definition of emergence as non-deducibility, belong to two different epistemological levels which have no direct connection and which are described by different models, irreducible to each other. It is in this lack of connection that the main problems related to the concept of emergence reside. A direct relationship would imply the possibility to describe two 
levels, and the respective domains, through a single model, so contradicting the definition of emergence as non-deducibility.

This epistemological problem can be solved if the difference in the behavior of the components in the system and in isolation-which we refer to as a downward causation-is ascribed to the observer's operations. In order to do so it is necessary to consider this difference as a difference between two distinct levels and domains of components which can be identified through two inequivalent operations of distinction: the material parts and the functional components. Therefore, downward causation can be considered as dependent on the different directions of the operations of distinctiontop-down for the functional components and bottom-up for the material parts-instead of a whole-parts interaction. According to this shifting of point of view and to the epistemological framework proposed here, it can be redefined as the irreducible difference between the bottom-up and top-down approaches: If there is a descriptive gap between levels due to a relation of non-deducibility, these two approaches cannot be considered the inverse of one another (Bich 2008, 2009). They therefore specify two different domains of components, the material structure and the organization, the latter being specified together with the unity it belongs to.

The irreducible gap between levels, in fact, makes the lower one not pertinent for the description of the others and so makes it impossible to establish a correspondence between levels. In such a scenario Kim's over-determination argument fails to be true. This is due to the role of the distinction between the different descriptive domains, which makes downward causation something different from a causal relationship. The epistemic shift between parts and components that characterizes it, in fact, does not allow us to express the higher level as an epiphenomenon. The reason is that it cannot be described exhaustively by models built on the properties of its material constituents. Consequently, from the epistemological point of view proper of this approach, if we focus the attention on the cognitive domain of the observer the whole is not already determined at the level of its material parts like in phenomena of pattern generation, and therefore there is no risk of over-determination.

\section{Conclusive remarks}


A particular idea of living system derives from this definition of biological autonomy in light of the concept of emergence. This is the idea of a unity that is never definable at only one level; a unity that always involves its generative dynamics although being at the same time irreducible to it. It is characterized by the continuous maintenance of a relational order which is invisible in its constituents, but nevertheless can be traced in the shape assumed by the interactions they instantiate in an abstract domain of relations. At the same time it is a unitary order which is generative of a further co-evolutive dynamics which takes place at a higher level through a systemic coupling with the environment from which it self-distinguishes.

These multilevel relations, characterized by the establishing of distinct and nonintersecting descriptive domains, bring forth an emergentist scenario which has important implications for the scientific practice. It consists in an anti-foundational perspective where there is neither a primitive level of description nor an objective reality to appeal to, but just an activity of construction of models that are limited in time and in the domain of application. This activity moves on manifold levels, which are distinct but at the same time all necessary in order to attain an understanding of the phenomena under study. It is a "dialectic without synthesis" between complementary descriptive levels considered in a relationship of mutual specification (Varela 1979). As such, it entails a shift in the use of descriptive models, from the extensional one typical of a reductionist framework to a dynamical one (Minati and Pessa 2006) — characterized by the interaction between different modalities of description - which seems suitable in order to catch the specificities of complex systems, especially in the biological domain.

\section{References}

Abel, D. L. \& Trevors, J. T. (2006). Self-organization vs. self-ordering events in lifeorigin models. Physics of Life Reviews, 3, 211-228

Anderson, P. W. \& Stein, D. L. (1985). Broken symmetry, emergent properties, dissipative structures, life: Are they related? (In E. F. Yates (Ed.), Self- 
organizing systems: The emergence of order (pp. 445-458). New York: Plenum Press.)

Banzhaf, W. (2002). Self-organizing systems. (In R. Mayers (Ed.), Encyclopedia of physical science and technology, vol. 14 (pp. 589-598). New York: Academic Press.)

Beer, S. (1980). Preface. (In H. Maturana \& F. Varela, Autopoiesis and cognition. The realization of the living (pp. 63-72). Dordrecht: Reidel Publishing.)

Bedau, M. (2003). Downward causation and autonomy in weak emergence. Principia, 6, $5-50$

Bedau, M. (2008). Is weak emergence just in the mind? Minds \& Machines, 18, 443459

Bich, L. (2008). L'ordine invisibile: Ripensare il vivente oltre la metafora computazionale. Ph.D. Dissertation, University of Bergamo

Bich, L. (2009). Downward causation and relatedness in emergent systems: Epistemological remarks. (In G. Minati, E. Pessa, \& M. Abram (Eds.), Processes of emergence of systems and systemic properties: Towards a general theory of emergence (pp. 591-601). Singapore: World Scientific.)

Bich, L. \& Damiano, L. (2007). Theoretical and artificial construction of the living: Redefining the approach from an autopoietic point of view. Origins of Life and Evolution of the Biosphere, 34, 459-464

Bich, L. \& Damiano, L. (2008). Order in the nothing: Autopoiesis and the organizational characterization of the living (In I. Licata \& A. Sakaji (Eds.), Physics of emergence and organization - Electronic journal of theoretical physics, Special Issue 2007 (pp. 343-373). Singapore: World Scientific.)

Bitbol, M. (2007). Ontology, matter and emergence. Phenomenology and the Cognitive Science, 6, 293-307

Ceruti, M. (1989). La danza che crea. (Milano: Feltrinelli)

Crutchfield, J. P. (1994). Is anything ever new? Considering emergence. (In G.A. Cowan, D. Pines, \& D. Meltzer (Eds.), Complexity: Metaphors, models and reality (pp. 515-533). Redwood City: Westview Press.)

Damiano, L. (2009). Unità in dialogo. (Milano: Bruno Mondadori Editore) 
Emmeche, C., Køppe, S., \& Stjernfelt, F. (2000). Levels, emergence, and three versions of downward causation. (In P. B. Andersen, C. Emmeche, N. O. Finnemann, \& P. V. Christensen (Eds.). Downward causation: Minds, bodies and matter (pp. 13-34). Århus: Århus University Press.)

Engels, F. (1952). Dialektik der Natur. (Berlin: Dietz Verlag)

Humphreys, P. (1997). How properties emerge. Philosophy of Science, 64, 1-17

Kim, J. (1992). 'Downward causation' in emergentism and nonreductive physicalism. (In A. Beckermann, H. Flohr, \& J. Kim (Eds.), Emergence or reduction? Essays on the prospects of nonreductive physicalism (pp. 119-138). Berlin: de Gruyter.)

Kim, J. (1993). Supervenience and mind: Selected philosophical essays. (Cambridge: Cambridge University Press)

Kim, J. (1997). Explanation, prediction and reduction in emergentism. Intellectica, 25, $45-57$

Kim, J. (1998). Mind in a physical world. (Cambridge, MA: MIT Press)

Lloyd Morgan, C. (1923). Emergent evolution. (London: Williams and Norgate)

Lyon, P. (2006). The biogenic approach to cognition. Cognitive Process, 7, 11-29

Maturana, H \& Varela, F. (1980). Autopoiesis and cognition: The realization of the living. (Dordrecht: Reidel Publishing)

Maturana, H. \& Varela, F (1984). El árbol del conocimiento. (Santiago del Chile: Editorial Universitaria)

Minati, G. \& Pessa, E. (2006). Collective beings. (New York: Springer)

Nicolis, G. \& Prigogine, I. (1977). Self-organization in nonequilibrium systems. (New York: John Wiley)

Pessa, E. (1998). Emergence, self-organization, and quantum theory. (In G. Minati (Ed.), First Italian conference on systemics (pp. 59-80). Milano: Apogeo)

Piaget, J. (1967). Biologie et connaissance. (Paris: Gallimard)

Prigogine, I. \& Stengers, I. (1979). La nouvelle alliance: Métamorphose de la science. (Paris: Gallimard)

Rosen, R. (1958). A relational theory of biological systems. Bulletin of Mathematical Biophysics, 20, 245-260

Rosen, R. (1978). Fundamentals of measurement and representation of natural systems. (New York: North-Holland) 
Rosen, R. (1991). Life itself: A comprehensive inquiry into the nature, origin, and fabrication of life. (New York: Columbia University Press)

Silberstein, M. \& McGeever, J. (1999). The search for ontological emergence. Philosophical Quarterly, 49, 182-200

Spencer Brown, G. (1969). Laws of form. (London: George Allen and Unwin Ltd)

Varela, F. (1979). Principles of biological autonomy. (New York: North-Holland)

Varela, F., Maturana, H., \& Uribe, R. (1974). Autopoiesis: The organization of living systems, its characterization and a model. Biosystems, 5, 187-196

Varela, F., Thompson, E., \& Rosh, E. (1991). The embodied mind: Cognitive mind and human experience. (Cambridge MA: MIT Press)

Waddington, C. H. (1968). The basic ideas of biology. (In C. H. Waddington (Ed.), Towards a theoretical biology: Prolegomena (pp. 1-41). Chicago: Adline.)

Weiss, P. (1969). The living system: Determinism stratified. (In A. Koestler \& J. R. Smythies (Eds.), Beyond reductionism: The Alpbach symposium (pp. 3-55). London: Hutchinson \& Co.)

Wiener, N. (1948). Cybernetics: Or control and communication in the animal and in the machine. (Cambridge, MA: MIT Press) 